\title{
Le rôle des chocs technologiques mondiaux dans les fluctuations agrégées
}

\author{
Martial Dupaigne $^{*} \quad$ Patrick Fève $^{\dagger}$
}

30 août 2004

\begin{abstract}
Résumé
Cet article étudie dans un contexte multinational l'évaluation fournie par Galí [1999] des effets des chocs technologiques et de leur contribution au cycle économique. Dans une premier temps, nous montrons que la méthodologie employée par Galí identifie correctement les chocs technologiques dans une économie autarcique. En revanche, si la taille de l'économie nationale est faible par rapport à celle du reste du monde et/ou les chocs étrangers sont plus volatils que les chocs nationaux, les résultats de Galí ne permettent pas de rejeter un modèle structurel à prix flexibles. Dans un second temps, nous montrons que, parmi les pays du G7, plus un pays est grand/fermé, plus les réponses estimées sont en accord avec celles du modèle théorique, de même que lorsqu'on considère le G7 en tant que zone.
\end{abstract}

Mots-clés : Chocs technologiques, Fluctuations agrégées, Économie ouverte.

Classification JEL : E32, F41.

\section{Introduction}

L'article de Galí [1999] a stimulé un renouveau du programme de recherches quant à l'évaluation de la contribution des chocs technologiques aux fluctuations agrégées. Cette littérature utilise des formes réduites multi-variées et des restrictions de long terme, cohérentes avec les enseignements théoriques, pour identifier empiriquement les chocs technologiques et leurs effets. Les résultats de Galí [1999] pour les États-Unis sont les

*GREMAQ, Université de Toulouse I, 21 allée de Brienne, F-31000 Toulouse, France. E-mail : martial.dupaigne@univ-tlse1.fr.

†GREMAQ, CNRS, IDEI et Banque de France. E-mail : patrick.feve@univ-tlse1.fr. Nous remercions Paul Beaudry, Fabrice Collard, Alain Guay, Franck Portier et Harald Uhlig pour leurs nombreux commentaires et suggestions. Nous restons seuls responsables d'éventuelles erreurs et omissions. Cet article reflète les idées personnelles des auteurs et n'exprime pas nécessairement la position de la Banque de France. 
suivants : i) les heures travaillées diminuent durablement après un choc technologique positif; ii) les chocs technologiques ne jouent qu'un faible rôle dans les fluctuations observées du produit et de l'emploi. Ces observations jettent le doute sur les modèles de cycle à prix flexibles, qui prédisent d'une part une hausse des heures travaillées à la suite d'un choc de productivité favorable, et d'autre part une influence prépondérante des impulsions d'origine technologique (Francis et Ramey [2002]).

Dans ce papier, nous évaluons les effets des chocs technologiques dans un contexte multinational.

Dans un premier temps, nous montrons en utilisant un modèle théorique pour engendrer des données que la méthodologie employée par Galí identifie correctement les chocs technologiques dans une économie autarcique à prix flexibles. En revanche, si la taille de l'économie nationale est faible par rapport à celle du reste du monde, et/ou si les chocs étrangers sont plus volatils que les chocs nationaux, cette procédure identifie mal les impulsions technologiques ${ }^{1}$. Cela signifie que, lorsque l'une de ces conditions est remplie, les résultats de Galí [1999] ne permettent pas de rejeter un modèle structurel reposant sur la flexibilité des prix.

Dans un second temps, nous confrontons les prédictions du modèle multinational aux observations des pays du G7. Nous montrons que plus un pays est grand/fermé, plus les réponses estimées au choc qui influence la productivité du travail à long terme sont en accord avec un modèle à impulsion technologique et prix flexibles.

\section{Le cadre d'analyse}

Nous présentons un modèle multi-pays soumis à différents chocs technologiques, commun et idiosyncratiques, que nous utiliserons comme processus générateur de données.

\subsection{Un modèle théorique multinational}

Nous considérons un modèle dynamique d'équilibre général à prix flexibles à la Baxter et Crucini [1993]. $n$ pays composent l'économie mondiale. La part de chaque pays dans la population totale est notée $\pi_{i}$, et $\sum_{i=1}^{n} \pi_{i}=1$. Dans ce qui suit, toutes les variables sont exprimées par tête.

\footnotetext{
${ }^{1}$ Précisément, la réponse estimée de l'emploi à l'innovation affectant à long terme la productivité du travail est négative alors que la réponse théorique de l'emploi national au choc technologique national est positive.
} 


\section{Les ménages}

Les ménages à durée de vie infinie résidant dans le pays $i$ reçoivent en dotation une unité de temps à chaque date. Ils choisissent leur plan de consommations et de loisirs, notées, $\left\{C_{i, t}\right\}_{t=0,+\infty}$ et $\left\{L_{i, t}\right\}_{t=0,+\infty}$, de manière à maximiser leur bien-être intertemporel anticipé :

$$
E_{0} \sum_{t=0}^{+\infty} \beta^{t} \frac{\left(C_{i, t}^{\theta} L_{i, t}^{1-\theta}\right)^{1-\sigma}}{1-\sigma}, i=1, \ldots, n
$$

en respectant les contraintes budgétaires et de temps énoncées par la suite.

\section{Les entreprises}

Les entreprises de l'ensemble des pays produisent le même bien à l'aide d'une technologie à rendements d'échelle constants, décrite par la fonction de production suivante

$$
Y_{i, t}=\left(\Gamma_{i, t} N_{i, t}\right)^{\alpha} K_{i, t}^{1-\alpha}, i=1, \ldots, n
$$

avec $0<\alpha<1$. Naturellement, $Y_{i, t}$ désigne dans cette expression le revenu dans le pays $i$ à la date $t, K_{i, t}$ et $N_{i, t}$ les quantités de facteur capital et travail utilisées et $\Gamma_{i, t}$ constitue le progrès technique portant sur le travail.

Le travail étant immobile, la quantité de travail employée dans chaque pays et à chaque date est soumise à la contrainte

$$
1-L_{i, t}-N_{i, t} \geq 0, i=1, \ldots, n \text {. }
$$

En revanche, le capital physique est un facteur de production mobile entre pays, mais dont l'accumulation est soumise à des coûts d'ajustement de la forme suivante :

$$
K_{i, t+1} \leq(1-\delta) K_{i, t}+I_{i, t}-\frac{\varphi}{2}\left[\frac{I_{i, t}}{K_{i, t}}-\left(\overline{\frac{I_{i, t}}{K_{i, t}}}\right)\right]^{2} K_{i, t}, i=1, \ldots, n \text { avec } \varphi>0 .
$$

Ici, $\left(\overline{\frac{I_{i, t}}{K_{i, t}}}\right)$ désigne le taux d'accumulation constant suivi le long du sentier de croissance équilibrée. Par conséquent, ces coûts d'ajustement s'annulent le long de ce sentier.

\section{Les chocs}

Afin de répliquer les estimations menées par Galí [1999], le processus suivi par la productivité du travail doit être intégré d'ordre un. Si les différents pays composant notre économie étaient frappés par des chocs technologiques I(1) indépendants, le modèle n'admettrait pas de croissance équilibrée. Pour cela, il s'avère nécessaire que les chocs nationaux suivent une tendance stochastique commune. Par conséquent, nous supposons que 
les productivités nationales évoluent selon le processus suivant

$$
\left\{\begin{aligned}
\Gamma_{i, t} & =A_{t} \exp e_{i, t}, \text { où } e_{i, t}=\rho e_{i, t-1}+\varepsilon_{i, t}, i=1, \ldots, n \\
A_{t} & =A_{t-1} \exp a_{t}
\end{aligned}\right.
$$

avec $0 \leq \rho<1$. Les $\left\{\varepsilon_{i, t}\right\}$ désignent $n$ bruit blancs de variance $\sigma_{\varepsilon_{i}}^{2}$ et $\left\{a_{t}\right\}$ est iid d'espérance $\bar{a}>1$ et de variance $\sigma_{a}^{2}$. Ainsi, la structure stochastique du modèle est composée de $n$ chocs nationaux transitoires et d'un choc agrégé permanent, portant tous sur la productivité des facteurs. ${ }^{2}$

\section{Les marchés}

L'unique bien final est parfaitement mobile, et la contrainte de ressources agrégée s'écrit à chaque date

$$
\sum_{i=1}^{n}\left(Y_{i, t}-C_{i, t}-I_{i, t}\right) \geq 0
$$

Les agents ont accès à un ensemble complet de titres contingents. L'équilibre décentralisé de cette économie est donc équivalent à la solution du programme centralisé suivant :

$$
\begin{aligned}
\max _{\left\{C_{i, t}, I_{i, t}, L_{i, t}, N_{i, t}\right\}_{t=0,+\infty}} & \mathcal{L}=E_{0} \sum_{t=0}^{+\infty} \beta^{t}\left\{\sum_{i=1}^{n} \pi_{i} \frac{\left(C_{i, t}^{\theta} L_{i, t}^{1-\theta}\right)^{1-\sigma}}{1-\sigma}\right. \\
& +\sum_{i=1}^{n} \Lambda_{i, t} \pi_{i}\left[(1-\delta) K_{i, t}+I_{i, t}-\frac{\varphi}{2}\left(\frac{I_{i, t}}{K_{i, t}}-\frac{\bar{I}_{i}}{K_{i}}\right)^{2} K_{i, t}-K_{i, t+1}\right] \\
& +\sum_{i=1}^{n} W_{i, t} \pi_{i}\left(1-L_{i, t}-N_{i, t}\right) \\
& \left.+P_{t}\left[\sum_{i=1}^{n}\left(Y_{i, t}-C_{i, t}-I_{i, t}\right)\right]\right\}
\end{aligned}
$$

\section{2 Étalonnage et protocole expérimental}

L'étalonnage des valeurs des paramètres structurels, présentées dans le tableau 1 , reprend des travaux précédents sur les États-Unis.

Le taux d'escompte psychologique est choisi de manière à ce que le rendement annuel du capital à l'état stationnaire soit de 6.5\%. Suivant Heathcote et Perri [2002], nous fixons l'élasticité intertemporelle de substitution $\frac{1}{\sigma}$ à .5 , et le paramètre $\theta$ est fixé de manière à ce que chaque individu travaille $30 \%$ de son temps disponible. L'élasticité de la production

\footnotetext{
${ }^{2}$ Il est possible d'introduire d'autres chocs (fiscaux ou sur les préférences) ayant les mêmes effets sur les variables domestiques que les chocs technologiques étrangers.
} 


\begin{tabular}{cccccccc}
\multicolumn{1}{c}{ TAB. 1 - Valeurs des paramètres } \\
\hline \hline$\beta$ & $\theta$ & $\sigma$ & $\alpha$ & $\delta$ & $\bar{a}$ & $\varphi$ & $\rho$ \\
\hline .99 & .34 & 2 & .58 & .025 & 1.004 & .1 & .9 \\
\hline \hline
\end{tabular}

au travail $\alpha$, le taux de dépréciation du capital physique $\delta$ et le taux de croissance moyen de la productivité globale des facteurs prennent respectivement les valeurs $0.58,0.025$ et 1.004 (voir Cooley et Prescott [1995]). Le coefficient du coût d'ajustement $\varphi=.1$ implique que l'investissement est environ trois fois plus volatil que le produit. Enfin, nous fixons à .9 le coefficient autorégressif des écarts nationaux de la productivité à la tendance commune mondiale, $\rho$. Les volatilités relatives des différents impulsions seront précisées dans chaque expérience.

\section{La procédure d'évaluation}

Pour ces valeurs des paramètres, nous résolvons par approximation numérique les conditions d'équilibre de l'économie. À partir du système espace-état solution, nous commentons dans un premier temps les réponses théoriques des variables endogènes du modèle à trois types de chocs : le choc technologique commun à l'ensemble des pays; le choc technologique national, qui est transitoire; et finalement, un choc technologique transitoire affectant les $n-1$ pays étrangers (ce qui serait "le" choc étranger dans un monde à deux pays).

Dans un second temps, nous tirons un vecteur d'impulsions et produisons les séries artificielles correspondantes. Sur ces séries, nous répliquons la méthodologie proposée par Galí [1999] qui utilise un modèle multivarié de type vectoriel auto-régressif (VAR) pour identifier le choc technologique. Ce modèle admet une représentation moyenne mobile infinie exprimant les taux de croissance ${ }^{3}$ de la productivité moyenne du travail $\Delta x_{t}$ et de l'emploi $\Delta n_{t}$ comme une somme de retards des deux impulsions $\left\{\varepsilon_{t}^{z}\right\}$ et $\left\{\varepsilon_{t}^{m}\right\}$

$$
\left[\begin{array}{l}
\Delta x_{t} \\
\Delta n_{t}
\end{array}\right]=\left[\begin{array}{ll}
C^{11}(L) & C^{12}(L) \\
C^{21}(L) & C^{22}(L)
\end{array}\right]\left[\begin{array}{c}
\varepsilon_{t}^{z} \\
\varepsilon_{t}^{m}
\end{array}\right] .
$$

Ces deux impulsions sont orthogonales, et on impose de plus la restriction identifiante $C^{12}(1)=0$, ce qui signifie que le second choc n'a aucun effet à long terme sur le taux de croissance de la productivité. Le premier choc, $\left\{\varepsilon_{t}^{z}\right\}$, est donc interprété comme "technolo-

\footnotetext{
${ }^{3}$ La modélisation des mouvements de basse fréquence de l'emploi est l'un des choix techniques faisant débat dans cette littérature (voir Christiano, Eichenbaum et Vigfusson [2003]). Nous avons adopté trois spécifications : taux de croissance et écarts à une tendance linéaire, comme Galí [2004]; niveaux, comme Christiano et al. [2003] et comme le suggère le modèle théorique. Les résultats qui ne sont pas reproduits ici par manque de place sont disponibles sur demande.
} 
gique" et le second, $\left\{\varepsilon_{t}^{m}\right\}$, comme "non-technologique" 4 . Nous présentons dans la section suivante les fonctions de réponse et les contributions des chocs à la variance de l'erreur de prévision, ainsi que des intervalles de confiance au seuil de $5 \%$ construits par simulations de Monte-Carlo.

\section{Enseignements du modèle structurel et application}

En utilisant le modèle théorique développé dans la section précédente comme processus générateur de données, nous montrons dans cette section que les erreurs d'identification liées à la restriction de long terme employée par Galí [1999] augmentent avec la dimension internationale de l'économie. Nous confrontons ensuite ces prédictions aux données de pays du G7.

\subsection{Les résultats}

\section{L'autarcie}

Nous considérons pour commencer une économie autarcique, c'est-à-dire $\pi_{1}=1$ (et donc nécessairement $\left.\pi_{2}=\ldots=\pi_{n}=0\right)$.

Dans le modèle théorique, une réalisation positive du choc technologique permanent (respectivement transitoire) entraîne une hausse persistante (respectivement temporaire) de la productivité, et conjointement une hausse temporaire de l'emploi ${ }^{5}$.

Les résultats du modèle VAR, présentés dans le cadran Nord-Ouest de la figure 1, montrent l'aptitude de la procédure de Galí [1999] à capturer les effets de ces chocs : la réponse observée de l'emploi au choc qui exerce un impact permanent sur la productivité est positive, et décroît au cours du temps. Ce choc explique la quasi-totalité de la variance de l'erreur de prévision sur la productivité, à tous les horizons, et entre 40 et $100 \%$ de la variance instantanée pour l'emploi.

\section{Taille et volatilité relative de l'économie nationale}

Lorsque l'on s'éloigne suffisamment de l'autarcie, un choc positif sur la productivité des pays étrangers entraîne une hausse durable de la productivité du travail et une baisse de l'emploi. En effet, les agents nationaux détiennent des titres émis par les firmes étrangères dont la valeur augmente à la suite du choc technologique favorable. Par un effet richesse,

\footnotetext{
${ }^{4}$ Dans un monde engendré par le modèle structurel, ce choc identifierait une combinaison linéaire des innovations des chocs stationnaires nationaux.

${ }^{5} \mathrm{La}$ substitution intertemporelle explique cette réponse de l'emploi lorsque le choc technologique est stationnaire.Lorsque le choc est permanent, l'emploi augmente pour permettre de retrouver le sentier de croissance équilibré (stochastique) en accumulant plus de capital.
} 
FIG. 1 - Fonctions de réponse empiriques au choc affectant la productivité à long terme et contributions à la variance de l'erreur de prévision (VEP)

Autarcie
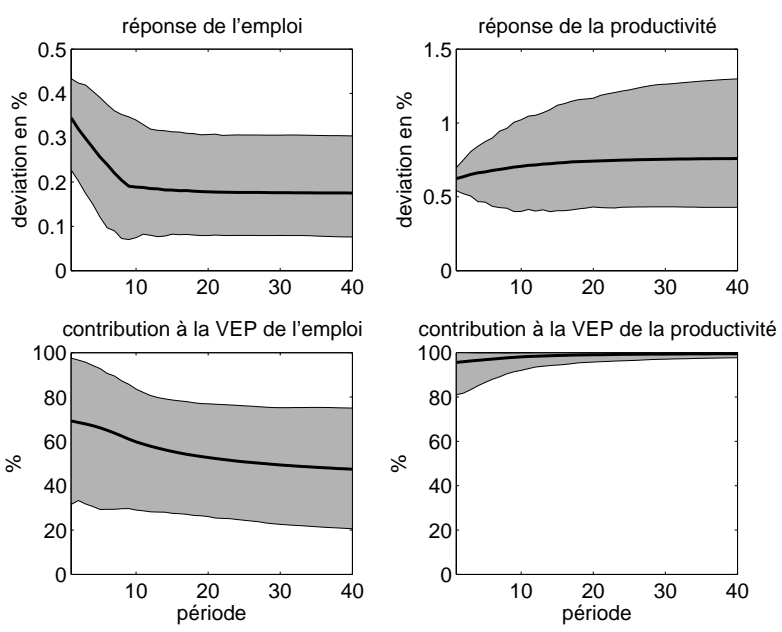

Reste du monde volatil
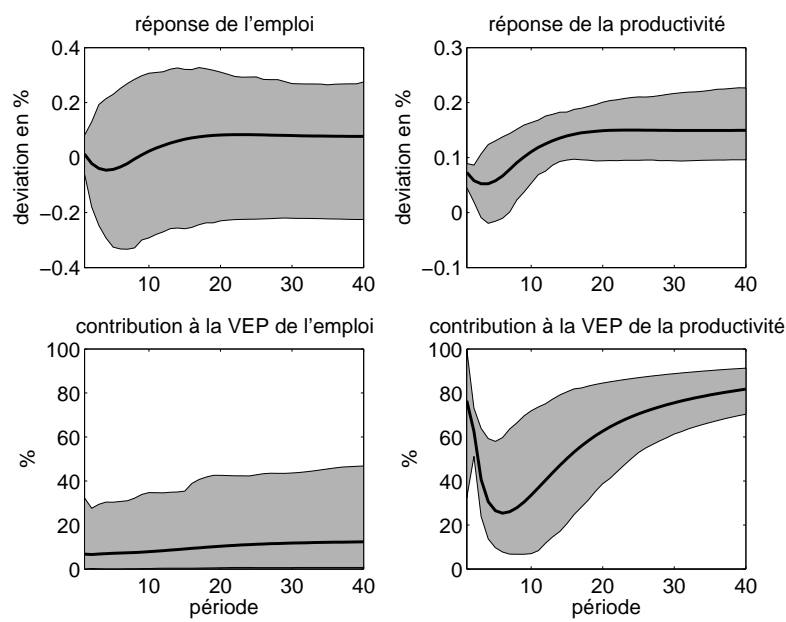

\section{Petit pays}
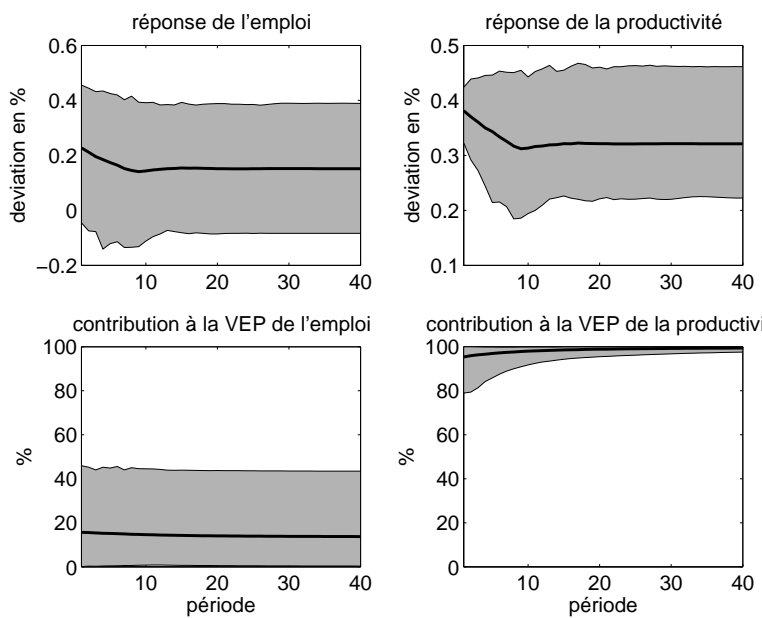

contribution à la VEP de la productivité

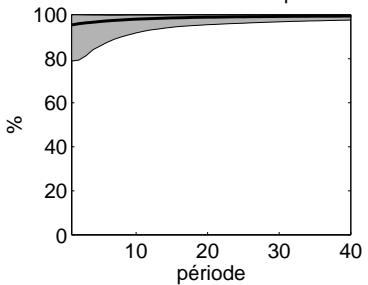

\section{Zone}
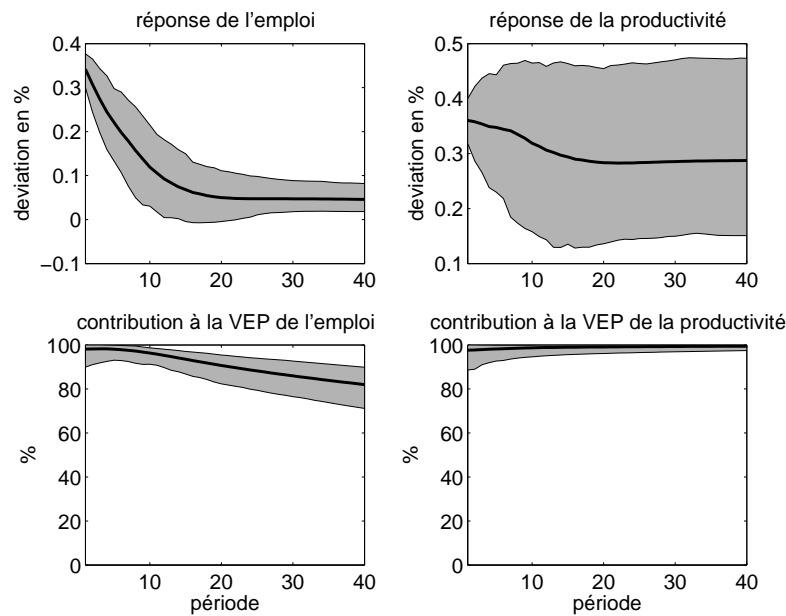

Note: le modèle VAR est estimée sur données issues du modèle multinational pour l'étalonnage du tableau 1. 250 simulations d'un échantillon de taille 200 ont été menées. 
l'offre de travail nationale baisse, d'où la réduction instantanée de l'emploi. Les chocs nationaux étant autocorrélés, la hausse courante de la productivité étrangère entraîne une réallocation progressive du capital physique au détriment de l'économie nationale qui déprime elle aussi l'emploi national.

Le cadran Nord-Est de la figure 1 décrit un pays représentant $5 \%$ de la population totale. Par rapport à l'autarcie, la réponse estimée de l'emploi au choc affectant la productivité de façon permanente est plus faible : il n'est désormais plus possible de rejeter une réponse nulle, à chaque date. La contribution de ce choc à la variance de l'erreur de prévision de l'emploi diminue elle aussi fortement par rapport à l'autarcie.

Le cadran Sud-Ouest de la figure 1 présente les réponses estimées lorsque l'écart-type des chocs sur la productivité des pays étrangers est 10 fois supérieur à celui des chocs domestiques et du choc agrégé. On constate la baisse estimée de l'emploi domestique à la suite d'un choc faisant augmenter la productivité à long terme. Cette configuration permet de retrouver de façon fallacieuse les résultats apparemment paradoxaux de Galí [1999] et [2004].

\section{L'économie agrégée}

Plutôt qu'une collection d'économies individuelles reliées les unes aux autres, nous étudions désormais le comportement de la zone économique formée par l'économie agrégée. L'agrégation ne pose pas de problèmes de mesure, car le bien final produit dans chacune des économies est identique, et le travail supposé homogène.

Dans le modèle théorique, le choc technologique agrégé exerce un effet permanent sur la productivité de la zone - et il s'agit du seul. L'emploi de la zone augmente à la suite d'une réalisation positive de ce choc.

Les effets estimés sur données agrégées, pour le même processus générateur de données que dans le cas précédent, du choc identifié par la restriction de long terme apparaissent dans le cadran Sud-Est de la figure 1. La procédure empirique identifie à nouveau le choc structurel. Ce résultat n'est pas surprenant à la lumière du premier cas envisagé, dans la mesure où l'économie agrégée forme une autarcie .

\footnotetext{
${ }^{6}$ Les estimations sont ici plus précises que dans l'autarcie étudiée précédemment. En effet, même si le choc mondial $\{a\}$ est le seul des deux chocs structurels présents dans le modèle théorique à exercer un effet permanent sur la productivité du travail, le choc technologique national peut lui aussi engendrer des mouvements relativement durables de la productivité. En petit échantillon, il est difficile de distinguer ces deux processus lorsque le coefficient autorégressif $\rho$ est élevé. Dans la zone, les effets des deux chocs nationaux se compensent partiellement.
} 


\subsection{Application empirique : le G7}

Nous étudions dans cette partie trois économies : les États-Unis, qui nous servent de référence; la France, qui constitue à la fois une économie plus petite et dont la volatilité relative à ses partenaires est plus faible; et enfin le G7, qui englobe notamment ces deux pays. Les données trimestrielles que nous utilisons sont issues des Principaux Indicateurs Économiques de l'OCDE, et couvrent la période $1978: 2$ à $2003: 4$ pour la France et le G7, de $1960: 2$ à $2004: 1$ pour les États-Unis.

FIG. 2 - Effets sur l'emploi du choc affectant la productivité à long-terme
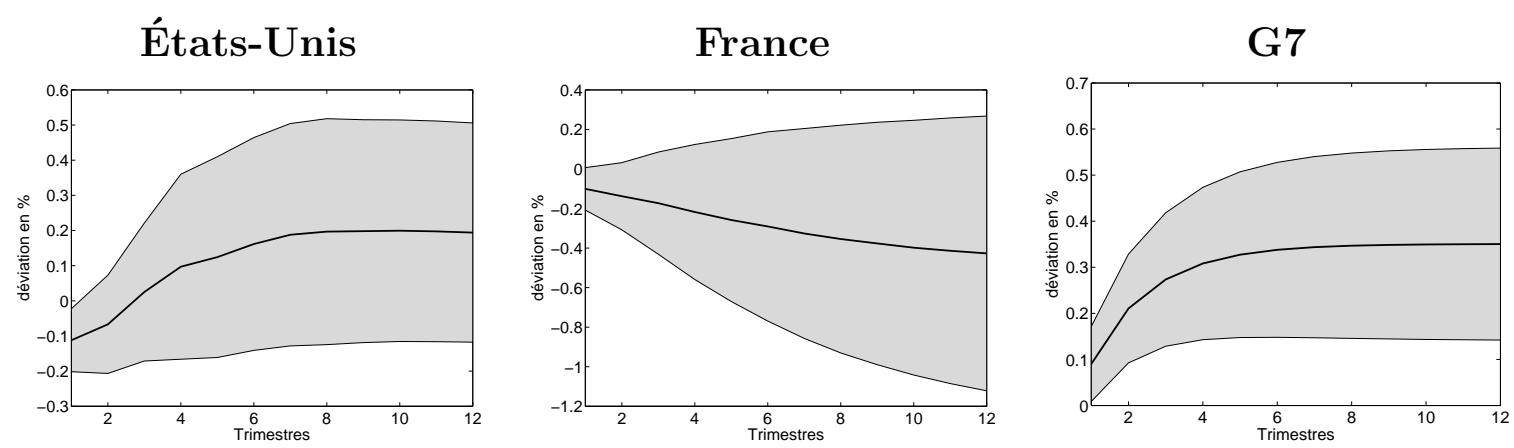

La figure 2 confirme les enseignements du modèle théorique :

i) Les réponses de l'emploi dans chacune des deux économies nationales prises individuellement sont négatives à court terme ${ }^{7}$. Il faut noter que les intervalles de confiance sont relativement importants si bien que seule la réponse à l'impact est significativement différente de zéro.

ii) La réponse de l'emploi au choc affectant la productivité à long terme est plus faible (plus négative) en France qu'aux États-Unis. De plus, l'impact négatif se limite au très court terme (trois trimestres) aux États-Unis, tandis que les effets négatifs sur l'emploi du choc technologique sont plus persistants en France.

iii) Sur la zone G7, l'emploi augmente instantanément et de façon persistante après un choc de productivité favorable. Cet effet positif est significatif quel que soit l'horizon. Cette plus grande précision des estimations est elle-même en accord avec les résultats des simulations effectuées à partir du modèle théorique (voir note 6).

\footnotetext{
${ }^{7}$ Cet effet négatif du choc affectant la productivité à long terme se retrouve pour les autres pays du G7, comme dans Galí [1999]. Ces résultats, non présentés ici, sont disponibles auprès des auteurs.
} 


\section{Conclusion}

Dans ce papier, nous montrons que l'évaluation de la contribution des chocs technologiques aux fluctuations proposée par Galí [1999] repose sur un modèle économétrique mal

spécifié. En dehors d'une situation autarcique, les chocs technologiques qui frappent les pays étrangers ont un impact négatif sur l'emploi national à travers un effet richesse, et donc un impact positif sur la productivité moyenne du travail. Lorsque les chocs de productivité nationaux sont persistants mais non permanents, la restriction de long terme utilisée ne permet pas dans un petit échantillon d'identifier correctement le choc domestique (qui entraîne une hausse de la productivité et de l'emploi domestique) d'un choc étranger (qui entraîne une hausse de la productivité domestique mais une baisse de l'emploi domestique). En d'autres termes, le choc faisant baisser l'emploi aux États-Unis identifié par Galí [1999] peut être une combinaison de chocs sur la productivité américaine et sur la productivité du reste du monde. Observer que l'emploi américain répond négativement à une telle impulsion ne permet pas, selon nous, de rejeter les modèles de cycles à prix flexibles.

\section{Références}

Baxter, M. et M. Crucini, Explaining saving investment correlation, American Economic Review, 1993, 83, 416-435.

Christiano, L. J., M. Eichenbaum, et R. J. Vigfusson, What happens after a technology shock?, Working Paper 9819, NBER 2003.

Cooley, T. et E. Prescott, Economic growth and business cycles, in T. Cooley, éditeur, Frontiers of Business Cycle Research, Princeton University Press, 1995, chapitre 1.

Francis, N. et V. A. Ramey, Is the technology-driven Real Business Cycle hypothesis dead? Shocks and aggregate fluctuations revisited, Working Paper 8726, NBER 2002.

Galí, J., Technology, employment and the business cycle : do technology shocks explain aggregate fluctuations?, American Economic Review, 1999, 89 (1), 249-271.

_ - On the role of technology shocks as a source of business cycles : some new evidence, Journal of the European Economic Association, 2004, 2 (2-3), 372-380.

Heathcote, J. et F. Perri, Financial autarky and international business cycle, Journal of Monetary Economics, 2002, 49, 601-627. 